\title{
The Informatics Olympiad in Mongolia: Training Resources for non-English Speaking Students
}

\author{
Altangerel KHUDER ${ }^{1}$, Danzan TSEDEVSUREN ${ }^{2}$ \\ ${ }^{1}$ School of Information, Communication Technology, Mongolian University \\ of Science and Technology \\ ${ }^{2}$ School of Mathematics and Natural Sciences, Mongolian National University of Education \\ e-mail:khuder@must.edu.mn, tsedevsuren@msue.edu.mn
}

\begin{abstract}
In this country report we present activities related to Mongolian team training for national and International Olympiads in Informatics. First we will outline the current conditions and problems Mongolian team is facing today. Then we describe possible solutions to those problems. At the end we will compare our achievements to our country report published in IOI conference in 2007. We hope this paper will be interesting for team leaders and teachers from non-English speaking countries.
\end{abstract}

Keywords: information and communication technology, informatics education, informatics competitions, programming contests, Informatics Olympiad, Mongolia.

\section{Introduction}

There are about 565 high schools and 497000 students in Mongolia (Purevjal and Altantuya, 2013). Each year Mongolian National Informatics Olympiad is organized in four stages: School competition, District competition, City/Province competition and National competition. Fig. 1. shows the participant numbers for each stage. Students with top scores are admitted to the next level.

The first National Informatics Olympiad was organized in 1987. Mongolian Informatics Association is responsible for organizing whole annual national informatics Olympiads in cooperation with the Ministry of Education and Science, other universities and ICT companies. In 2015 we've started using CMS (Contest Management System) in our National Competition which reduced the time needed for judging and made it less human dependable (Maggiolo, Mascellani, and Wehrstedt, 2014).

The first four winners of the national Olympiad participate in the International Olympiad in Informatics. The national winners receive scholarships to study IT at local universities (Choijoovanchig, Uyanga, and Dashnyam, 2007). 


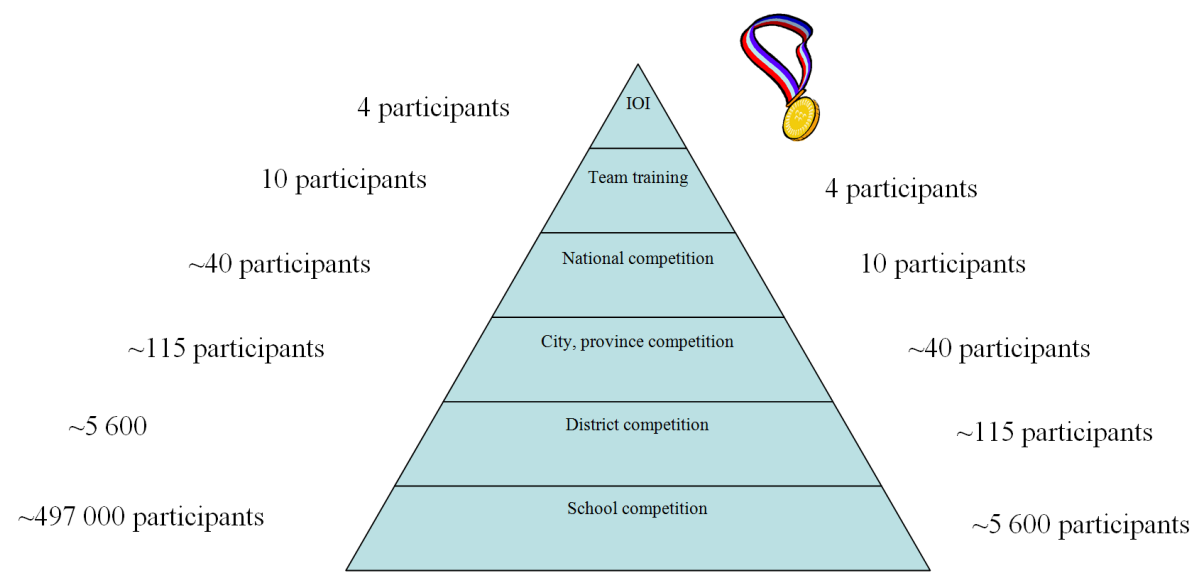

Fig. 1. Participants and structure of National Olympiad.

Professors from leading IT universities such as Mongolian National University of Education, Mongolian University of Science and Technology and National University of Mongolian, participate in trainings for IOI team.

Students solve 6 programming problems in two days for National competition. The maximum score at the competition is 300 or 600 points. Fig. 2 shows the total participant number and the percentage of the maximum score from absolute high score.

Fig. 3. shows results of the Mongolian team participation in IOIs from 2011 through 2015.

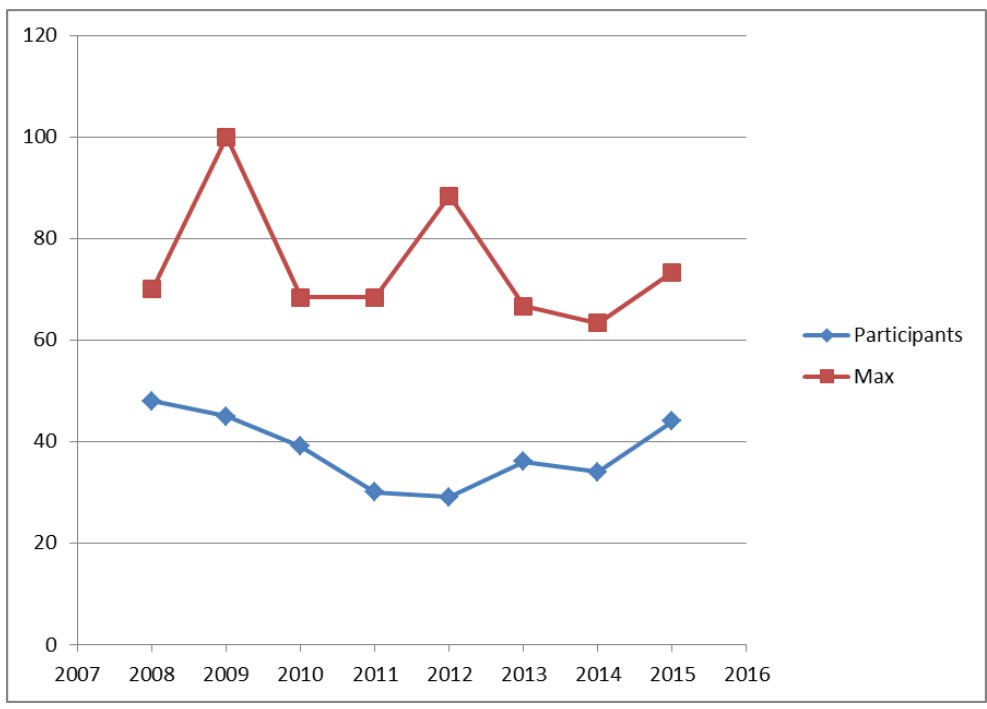

Fig. 2. Maximum score (in percentage from absolute high score) and participant number. 


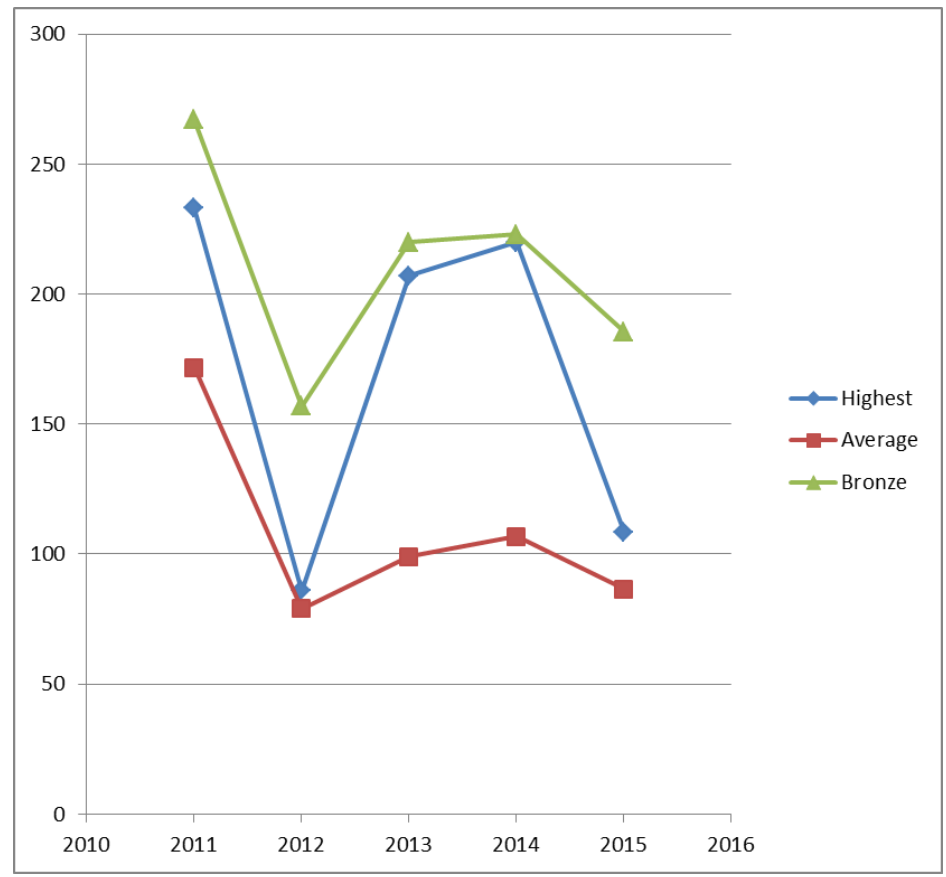

Fig. 3. Mongolian team results in IOI.

\section{Key Issues and Problems}

The following issues are the key challenges that hinder the success of Mongolian team at the international competition:

1. Weak English language. Due to language barriers students cannot fully understand tasks, use online internet sources and participate in online contests. Regular participation in online contests can be very useful for the development of students' programming and algorithmic skills.

In addition, online contest rankings show us the preparedness of our students at the international level.

2. Lack of student's skills and knowledge related to IOI syllabus. IOI syllabus gives us list of knowledge that is required to solve most of the IOI problems. Lack of knowledge on IOI syllabus results badly planned training and subsequently lack of success at IOI.

3. Lack of highly qualified teachers to train students. The most successive former IOI participants are going abroad after graduation to study and work. Now they work for Google, Microsoft, Facebook, Amazon etc. This kind of "brain drain" takes most of our teachers away. If former IOI participants would share their experience with new participants it could help them much.

In the next section we will list how we have tried to improve students' skills and knowledge of IOI. 


\section{Improvements}

Most of the problems stated in the previous section related to English language skills. Though teaching English is not our main purpose we've started looking for websites, online contests, which can support Mongolian language. To solve a problem student must translate the problem into Mongolian. However, it takes a lot of time and in addition student can misunderstand the problem.

There are many online contests organized almost every day on the internet. As most of our students are not strong in English we were interested in websites which allow us to add problems in Mongolian language and upload the tests or just translate the problems into Mongolian language.

So far we've found following websites and contests:

1. Bebras. Last year our students were able to do the Bebras problems in their native language and they were excited because there were enough problems to challenge themselves. In this contest we had wide choice for the easy and hard problems (Vegt, 2013). We think many high school students were stimulated to participate in informatics Olympiad after their Bebras participation.

2. SPHERE online judge (www.spoj.com). In 2008 one of our committee members contacted Andrew Kosowski from Gdansk University, Poland and asked permission to translate the problems on the website into Mongolian language. Now most of the basic level online problems in Mongolian language are on SPHERE. Now several teachers from Mongolian can add problems and organize contest on SPHERE servers.

3. Croatian Open Competition in Informatics (www.hsin.hr/coci). There are 7 online contests in a year and the organizers allow translators from other countries to get the problems in English and translate into their native language before each online contest.

4. Hackerrank. The website allows to its users to be problem setter and organize a contest.

5. USACO. One of our contestants contacted a personal from USACO and they agreed him to get problems before the contest and translate them into Mongolian.

6. Asia-Pacific Informatics Olympiad. The Asia-Pacific Informatics Olympiad (APIO) is an IOI-like competition for delegations within the South Asian / Western Pacific region.

All these websites and online resources became available for us thanks to international cooperation. Therefore we think one of the best ways to improve quality of our national team is international cooperation.

As a result of a fruitful cooperation with Russian team we got their full syllabus for IOI preparation. Now we have translated the syllabus in Mongolian language and we hope this will help us to organize well-planned training.

Recently some of the former contestants are coming back to Mongolia to share their experience with other contestants. That is a good way to share their knowledge with the next generation and this kind of feedback will help us to support continuous development of Mongolian team. 
Also we have a dedicated website for our National Olympiad in Informatics so we can save our results for later analysis (Mongolian National Olympiad NGO, 2015).

\section{Summary}

Compared to Mongolian report in 2007 there were following main improvements:

- We introduced full IOI syllabus to students.

- We have an official website for National contest.

- We started using IOI judge system in National contest (CMS).

Skills and knowledge of Mongolian students are improving each year and there are more and more students interested in Informatics Olympiad. To support them further we will need some text book in the future.

Another future work for us is to develop international cooperation further so that our students can participate in many more online contests in their native language. We hope this way they can achieve good placements in IOI and become good specialists.

\section{References}

Mongolian National Olympiad NGO (2015). (In Mongolian). Retrieved 2016.04.21 from: www. informatics.mn

Choijoovanchig, L., Uyanga, S., Dashnyam, M. (2007). The Informatics Olympiad in Mongolia. Olympiads in Informatics, 1, 31-36.

Maggiolo, S., Mascellani, G., Wehrstedt, L. (2014). CMS: a Growing Grading System. Olympiads in Informatics, 123-131.

Purevjal, A., Altantuya, Y. (2013). Statistical Report 2013. Ulaanbaatar: OchirPress LLC.

Vegt, W. v. (2013). Predicting the difficulty level of a Bebras task. Olympiads in Informatics, 132-139.
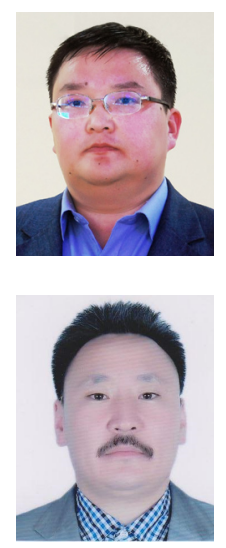

A. Khuder is an associate professor of the School of Information, Communication Technology, Mongolian University of Science and Technology. He is $\mathrm{PhD}$ in ICT and actively participates in the organization of Mongolian Informatics Association. His research interests include Natural Language Processing, Operating Systems and Artificial Intelligence.

D. Tsedevsuren is Professor at School of Mathematics and Natural Sciences, Mongolian National University of Education. $\mathrm{He}$ is $\mathrm{PhD}$ in ICT and Educational Studies, and he is currently working as a President of this Mongolian Informatics Association. His research interests include Informatics education, ICT in eduaction, theory and methodology of eLarning and electronic learning content development. 